This item was submitted to Loughborough's Research Repository by the author.

Items in Figshare are protected by copyright, with all rights reserved, unless otherwise indicated.

\title{
Rhétorique de la cohésion sociale et paradoxes de la « paix par le bas » en Côte d'Ivoire »
}

PLEASE CITE THE PUBLISHED VERSION

http://www.karthala.com/revue/2250-politique-africaine.html

PUBLISHER

Karthala

VERSION

AM (Accepted Manuscript)

\section{PUBLISHER STATEMENT}

This work is made available according to the conditions of the Creative Commons Attribution-NonCommercialNoDerivatives 4.0 International (CC BY-NC-ND 4.0) licence. Full details of this licence are available at: https://creativecommons.org/licenses/by-nc-nd/4.0/

\section{LICENCE}

CC BY-NC-ND 4.0

\section{REPOSITORY RECORD}

Piccolino, Giulia. 2019. "Rhétorique De La Cohésion Sociale Et Paradoxes De La « Paix Par Le Bas » En Côte D'ivoire »”. figshare. https://hdl.handle.net/2134/28457. 


\title{
Rhétorique de la cohésion sociale et paradoxes de la « paix par le bas " en Côte d’Ivoire
}

\author{
Giulia Piccolino \\ Loughborough University, Email: g.piccolino@lboro.ac.uk
}

Ce manuscrit est la version acceptée (avant les dernières révisions et la relecture typographique) d'un article à paraitre en 2018 dans la revue Politique Africaine.

This is the accepted manuscript (before last revisions and copy editing) of an article due to appear in 2018 on the journal Politique Africaine.

\begin{abstract}
Résumé
Après 2011, le gouvernement de la Côte d'Ivoire et la communauté internationale se sont engagés dans des actions de soutien à la paix au niveau local. Ces actions apparaissent cependant problématiques dans un contexte marqué par l'absence de réconciliation entre les élites nationales. Cet article, basé sur des recherches conduites à Abidjan et dans l'Ouest du pays, offre une réflexion critique sur la place des programmes de soutien à la paix au niveau local dans le modèle de reconstruction post-conflit du gouvernement Ouattara et interroge le grand principe qui les étaye: l'argument selon lequel le « local » constitue une sphère distincte du niveau national, alors même que les acteurs locaux entretiennent des relations importantes - tant matérielles que symboliques - avec les élites nationales.

\section{English abstract \\ Social Cohesion and the paradoxes of building peace from below in Côte d'Ivoire}

After 2011, the government of Côte d'Ivoire and the international community engaged in actions aimed at supporting peace at the local and community level. These actions appear however problematic in a political environment characterised by the lack of elite-level reconciliation. This article, based on research carried out in Abidjan and in the West of the country, reflects critically on the role that local peacebuilding programmes play within the model of post-conflict reconstruction put forward by the Ouattara government and challenges the assumption that the "local" might constitute a sphere separate from the national level. It highlights how local actors entertain important relationships - material but also symbolic - with national elites.
\end{abstract}

\section{Introduction}

Après l'arrivée au pouvoir d'Alassane Ouattara en 2011, le gouvernement Ivoirien insiste sur la nécessité de construire la paix entre les citoyens Ivoiriens. À la rhétorique de la réconciliation, qui a accompagné la création de la Commission Dialogue, Vérité et Réconciliation (CDVR) en 2011, s’est graduellement ajoutée celle de la « cohésion sociale », surtout après la création du Programme Nationale de Cohésion Sociale (PNCS) en 2012. La promotion de la cohésion sociale, qui inclut des actions de reconstruction d'infrastructures communautaires et de promotion du dialogue au niveau local, apparait le résultat de l'appropriation par le gouvernement Ivoirien de l'agenda de promotion de la paix au niveau locale de la communauté internationale. Celle-ci a dans les dernières années insisté sur l'importance de la dimension locale, suivant le paradigme dit du « local turn ${ }^{1} »$.

1. R. Mac Ginty et O.P. Richmond, « The Local Turn in Peace Building: a Critical Agenda for Peace », Third World Quarterly, vol. 34, n 5, 2013, p. 763-783. Voir aussi S. Autesserre, The Trouble with the Congo: Local Violence and the Failure of International Peacebuilding, Cambridge; New York, Cambridge University Press, 
Cependant, la construction de la paix au niveau local est particulièrement problématique dans le cas de la Côte d'Ivoire, où la paix actuelle au niveau national est basée sur une victoire militaire. Cet article s'interroge sur l'idée qu'une réconciliation entre communautés puisse être achevée dans un contexte de «paix des vainqueurs » et en l'absence de résolution du conflit entre les principaux acteurs politiques nationaux. Il montre qu'en Côte d'Ivoire la rhétorique de la cohésion sociale a été mise au service d'une vision dépolitisée de la construction de la paix et a été utilisée pour déplacer la responsabilité de la réconciliation des élites politiques nationales vers les communautés locales. Cependant, dans un contexte où les liens entre élites politiques et communautés sont très forts, le manque de réconciliation entre les élites se répercute aussi sur les communautés et compromet le succès des programmes censés promouvoir la « cohésion sociale ».

C'est cela qui ressort d'une série de focus groups conduits à Abidjan et dans l'Ouest du pays, dans lesquels nous avons demandé aux Ivoiriens de différentes couches sociales et affinité politique de parler de leurs priorités en termes de paix et des obstacles à la réconciliation. Même si elle a une longue tradition, le rôle de la méthodologie des focus groups dans le cadre de la recherche sur la paix et les conflits a été peu théorisée ${ }^{2}$. Par rapport aux objectifs de cet article, cette méthodologie offre deux avantages. Différemment que les sondages d'opinion, elle permet aux personnes qui devraient être les protagonistes des efforts de construction de la paix au niveau local de s'exprimer dans leurs propres mots et avec plus de liberté. Par rapport à l'entretien individuel, elle permet à ces personnes, qui souvent ne sont pas habituée à s'exprimer sur la situation du pays et discuter leurs opinions, de le faire dans un cadre qui leur est plus familiale et où il y a moins de pression.

La première section de cet article récapitule les circonstances de l'arrivée au pouvoir d'Alassane Ouattara en 2011, et le cadre général de la transition post-conflit de la Côte d'Ivoire. Dans la deuxième et troisième section de l'article j'analyse la politique de promotion de la réconciliation et de la cohésion sociale menée par le gouvernement et par les bailleurs de fonds. Dans la dernière section, je discute les attitudes par rapport à la paix des ceux qui devraient être les destinataires de cette politique - c'est-à-dire les citoyens qui ne font pas partie de l'élite politique et économique du pays. Dans la conclusion, j'analyse la situation de paix actuelle en Côte d'Ivoire et ses limites.

\section{La construction de la paix entre dimension locale et nationale}

La crise post-électorale de 2011-2012 a été l'étape finale d'une longue période d'instabilité politique en Côte d'Ivoire, qui remonte aux années $1990^{3}$. Avec l'arrivée au pouvoir d'Alassane Ouattara en 2011, la Côte d'Ivoire semble avoir retrouvé une relative stabilité. Cependant, les circonstances dans lesquelles cette stabilité a été obtenue posent des problèmes par rapport à la possibilité de tourner la page de l'histoire des divisions communautaires.

Ce qui caractérise la transition post-conflit en Côte d'Ivoire est le fait que la paix ne repose pas sur un processus de négociation, mais sur la victoire - militaire et électorale à la fois -

2010 et, pour une revue plus exhaustive de la literature, H. Leonardsson et G. Rudd, « The 'Local Turn’ in Peacebuilding: a Literature Review of Effective and Emancipatory Local Peacebuilding », Third World Quarterly, vol. 36, n 5, 2015, p. 825-839.

2. Pour une exception, voir J. Söderström, «Focus Groups: Safety in Numbers», in K. Höglund et M. Oberg (dir.), Understanding Peace Research: Methods and Challenges, Abingdon (UK), Routledge, 2011, p. 146-164. 3. F. Akindès, The Roots of the Military-Political Crises in Côte d'Ivoire, Uppsala, Nordiska Afrikainstitutet, 2004. Voir aussi C. Bouquet, Côte d'Ivoire: Le Désespoir de Kourouma, Paris, Armand Colin, 2011 et M. McGovern, Making War in Côte d'Ivoire, Chicago, University of Chicago Press, 2011 pour un cadre général de la crise Ivoirienne. 
d'un camp sur l'autre 4 . L'ancien président Gbagbo n'est plus considéré aujourd'hui comme un interlocuteur du processus de paix, comme il l'a été dans la période 2002-2010, mais plutôt comme un criminel de guerre présumé, qui a été arrêté et transféré à la Haye pour être jugé par la Cour Pénale Internationale (CPI). Plusieurs de ses partisans ont aussi été détenus pendant des périodes plus ou moins longues après $2011^{5}$.

Dans ce contexte de marginalisation de l'opposition, la transition post-conflit ivoirienne a été marquée par une vision particulière de la paix et de la reconstruction du pays, celle du président Ouattara et du Rassemblement des Houphouëtistes pour la Démocratie et la Paix (RHDP) qui le soutient. L'héritage de Félix Houphouët-Boigny, avec lequel le RHDP revendique une continuité politique, influence cette vision. Dans la rhétorique d'HouphouëtBoigny, un bon leadership, la prospérité économique et la paix était interconnectés ${ }^{6}$. Pour l'ancien président Ivoirien, la paix était le préalable du développement, et, à son tour, le développement renforçait la paix. L'accent mis sur la paix n’empêchait pas le régime d'Houphouët-Boigny d'être autoritaire et d'utiliser la violence contre ceux qui menaçaient le pouvoir du président. Cependant, dans la mesure où la contestation restait limitée et ne mettait pas en discussion le régime d'Houphouët-Boigny en tant que tel, le " dialogue » était accepté et même encouragé7.

L’idée qu'il existe un lien étroit entre développement et paix informe aussi la vision de Ouattara, renforcée dans son cas par sa formation d'économiste. Dans la philosophie de construction de la paix du régime Ouattara, la croissance économique du pays « guérira » les Ivoiriens de leurs traumatismes ${ }^{8}$. Par conséquent, les actions visant à relancer l'économie ivoirienne et attirer les investissements ont clairement été une priorité pour le nouveau régime $^{9}$. Le gouvernement Ouattara présente la Côte d'Ivoire non pas comme un pays postconflit ayant besoin d'une assistance, mais comme un pays « émergent » qui doit retrouver un rôle de leadership au plan régional.

Tandis que la stratégie économique du régime Ouattara a enregistré quelques succès, les efforts de dialogue entre le gouvernement Ouattara d'un côté, et le FPI et d'autres organisations pro-Gbagbo de l'autre ont manqué d'envergure et se sont heurtés à la mauvaise volonté des deux parties. Alassane Ouattara et le RHDP s'estiment politiquement trop forts pour faire des concessions aux anciens ennemis. L’héritage houphouëtiste et sa conception du dialogue ont aussi eu, de ce point de vue, un impact ambivalent sur l'attitude du gouvernement actuel. En lien avec la tradition Houphouetiste, le président Ouattara et ses alliés se sont, à certaines occasions, montrés disponibles à faire des concessions matérielles où à « pardonner » les membres du camp adverse qui se montrent disponibles à accepter des

\footnotetext{
4. G. Piccolino, « Peacebuilding and Statebuilding in Côte d'Ivoire under the Alassane Ouattara presidency. A victor's peace?», African Affairs, à paraitre.

5. M.K. Gbagbo, Rapport n.1-2017: interrogations sur la cohésion nationale [en ligne], 20 Janvier 2017 <http://lecombattant.canalblog.com/archives/2017/02/01/34877791.html>, consulté le 7 Mai 2017 ; Amnesty International, Côte d'Ivoire 2016/2017 [en ligne] <https://www.amnesty.org/fr/countries/africa/cote-divoire/report-cote-divoire/>, consulté le 7 Mai 2017.

6. J. Maddox Toungara, «The Apotheosis of Côte d'Ivoire's Nana Houphouët-Boigny », The Journal of Modern African Studies, vol. 28, nº 1, 1990, pp. 23-54.

7. J. Maddox Toungara, «The Apotheosis of Côte d'Ivoire’s Nana...», art. cité; C. Vidal, « La brutalisation du camp politique Ivoirien », Revue Africaine de Sociologie, vol. 7, n 2, pp. 45-57.

8. M. Ricard, « Gouverner le 'post-conflit' en Côte d'Ivoire », Annuaire Français de Relations Internationales, vol. 43, 2017; B. Charbonneau, "Côte d'Ivoire : possibilités et limites d'une réconciliation », Afrique contemporaine, vol. 1 , $n^{\circ} 245,2013$, pp. 111-129.

9. G. Piccolino, « Peacebuilding and Statebuilding in Côte d’Ivoire», art. cité.
} 
compromis $^{10}$, tels que le président officiel du FPI Pascal Affi N'Guessan, mais ils n'acceptent pas les arguments de l'opposition et ses revendications sur le plan politique et symbolique.

De l'autre côté, le camp Gbagbo a aussi peu de motivations à s'investir sérieusement dans le dialogue, étant donnée l'hégémonie actuelle du RHDP et de facto l'impossibilité d'une victoire électorale. Pendant plusieurs années le FPI, dont certains dirigeants étaient emprisonnés, est apparu enfermé dans une rhétorique jusqu'au-boutisme et victimaire. La sortie de prison du président du parti, Affi N’Guessan, et les élections présidentielles de 2015 ont compliqué la donne, en provoquant un schisme entre les partisans de Affi N'Guessan, qui s'est porté candidat aux élections, et la « vieille garde » du parti, guidée par le vice-président Aboudramane Sangaré, qui a persévéré dans le boycott.

Dans ce contexte, les attitudes de la population ivoirienne restent polarisées. Les résultats des élections présidentielles de 2015 ont confirmé qu'il y a en Côte d'Ivoire un "camp Gbagbo », représentant une portion considérable de l'électorat, qui se sent exclu du système politique $^{11}$. Si on compare ces résultats à ceux de 2010, on peut estimer que plus d'un million et demi d'anciens électeurs de Gbagbo, correspondant à peu près à un quart des électeurs inscrits, a boycotté les élections ${ }^{12}$.

Le gouvernement ivoirien se trouve donc dans une situation paradoxale. D’un côté, au niveau de la politique nationale, le président est solidement installé au pouvoir, l'opposition est affaiblie et la véritable compétition politique est à l'intérieur du RHDP. De l'autre côté, au niveau de la population, des divisions profondes demeurent, et la légitimité du régime RHDP est mise en question. Cette situation a poussé le régime Ouattara à s'engager dans une série d'activités censées promouvoir la «réconciliation» et la «cohésion sociale » entre les Ivoiriens.

Dans le paragraphe suivant, je reconstruis l'histoire de ces efforts. Je démontre comment le processus a été progressivement dépolitisé, et l'accent de plus en plus mis sur la dimension locale et communautaire, et sur la nécessité pour les citoyens Ivoiriens d'opérer pour la " cohésion sociale ", en se montrant prêts à dialoguer avec leurs voisins et à participer aux projets locaux animés par les ONGs et par le gouvernement. Cependant, au même temps leurs dirigeants politiques ne montrent pas de volonté sincère de s'engager de la même façon dans le dialogue et la réconciliation avec leurs adversaires.

\section{De la réconciliation nationale à la montée en puissance de la rhétorique de la cohésion sociale}

L'un des premiers et plus ambitieux efforts du gouvernement Ouattara en matière de construction de la paix a été la création de la CDVR en Mai 2011, juste après la fin de la crise post-électorale. Le mandat de la Commission incluait la recherche de la vérité, le dialogue et les auditions des victimes ${ }^{13}$. L'approche de la CDVR était donc holistique, car elle essayait de

10. Sur le rôle du pardon dans l’idéologie Houphouetiste voir J. Maddox Toungara, «The Apotheosis of Cote d'lvoire’s Nana...», art. cité; M. McGovern, Making War in Côte d'Ivoire, op. cit.

11. G. Piccolino, «One Step Forward, Two Steps Back? Côte d’Ivoire’s 2015 Presidential Polls», Africa

Spectrum, vol. 51, n01, 2016, pp. 97-110.

12. Calculs basés sur République de Côte d'Ivoire, Second tour de l'élection du Président de la République, Scrutin du 28 Novembre 2010, Commission Electorale Indépendante, et Abidjan.net, Election du Président de la République, Scrutin du 25 Octobre 2015, Résultats fournis par la Commission Electorale Indépendante [en ligne], 27 Octobre 2015 < http://abidjan.net/ELECTIONS/presidentielle/2015/resultats.html >, consulté le 11 Avril 2017.

13. D. Lopes, La Commission Dialogue Vérité et Réconciliation en Côte d'Ivoire: la réconciliation n'a pas eu lieu, Bruxelles, Groupe de Recherche et d’Information sur la Paix et la Sécurité (GRIP), 30 Juin 2015. 
cibler à la fois le niveau national, en reconstruisant l'histoire des crises politiques depuis 1990, et le niveau des communautés et des individus, en collectant les témoignages des leaders communautaires et des victimes de la crise, à travers ses bureaux régionaux.

Dès le début, la CDVR a montré des faiblesses. Le mandat de la Commission était excessivement ambitieux, en considérant aussi qu'elle n'était initialement nommée que pour deux ans. Le choix comme président de la Commission d'un acteur politique, l'ancien premier ministre Charles Konan Banny, s’est révélé questionnable. L’arrogance de Konan Banny et son incapacité de prendre en compte les préoccupations de la société civile, ainsi que ses ambitions politiques, ont compliqué les relations avec ses partenaires nationaux et internationaux ${ }^{14}$. Mais la CDVR s'est aussi heurté à l'attitude ambivalente du gouvernement ivoirien, qui, même s’il encourageait dans sa rhétorique le dialogue, n’était pas disposé à accepter que des critiques importantes à l'égard du leadership politique actuel émergent de ce dialogue. Finalement, la CDVR a réussi à auditionner 70000 personnes, inclue des victimes, des responsables de la violence politique, et des témoins. La plupart du travail de la CDVR n'a eu cependant que peu d'impact. À cause des querelles entre Charles Konan Banny et le gouvernement et de la réticence de ce dernier par rapport aux conclusions de la CDVR, par exemple, les audiences de la Commission n’ont pas été transmises par la télévision d’État. De façon similaire, le rapport final de la CDVR, présenté au président le 15 Décembre 2014, a été rendu public seulement deux ans plus tard. Les passages du rapport concernant les opinions des électeurs de Gbagbo sur les élections de 2010, et sur les responsabilités de Ouattara dans la crise, ont été jugés inacceptables par le gouvernement et ont était expurgés dans la version publié ${ }^{15}$.

C'est dans ces conditions d'impasse du processus entamé par la CDVR que l'attention du gouvernement s'est tournée de plus en plus vers le niveau local et communautaire. Ce développement a été accompagné par la montée en puissance d'un discours axé sur la cohésion sociale. Ce discours a été originairement diffusé en Côte d'Ivoire par la communauté internationale, mais le gouvernement Ivoirien l'a progressivement approprié, jusqu’à lancer un programme gouvernementale dédié à la « cohésion sociale ».

Le paradigme de la résolution de conflit au niveau local et de la cohésion sociale a ses racines dans la réflexion académique, où il a été discuté sous l'étiquette de local turn, et a été progressivement adopté par les institutions internationales dans la dernière décade ${ }^{16}$. L'influence de ce paradigme sur la politique des institutions internationales en Côte d'Ivoire est évidente, si on regarde une série de projets lancés par les partenaires déjà dans la période avant 2011, quand la Côte d'Ivoire était enlisée dans une situation ambiguë de « ni guerre ni paix ${ }^{17}$. Entre les activités inspirées par le paradigme du local turn on peut mentionner celles de la Division des Affaires Civiles (DAC) de l’Opération des Nations Unies en Côte d'Ivoire

14. Entretien avec dirigeant d'ONG, Abidjan, 4 Juin 2015.

15. C’est cela qui ressort en comparant un résumé confidentiel du rapport de la CDVR obtenu par l'autrice de cet article et le rapport publié : CDVR, « Rapport Final» [en ligne], République de Côte d’Ivoire, Abidjan, publié le 26 Octobre 2016 avec la date de Décembre 2014,

<https://www.gouv.ci/doc/presse/1477497207RAPPORT\%20FINAL_CDVR.pdf>, consulté le 13 Mars 2017. 16. Voir articles cités à la note 2. Sur la cohésion sociale, voir aussi F.D. Cox et T.D. Sisk (dir.), Peacebuilding in Deeply Divided Societies. Toward Social Cohesion? Basingstoke (UK), Palgrave McMillan, 2017. Par rapport à l'influence de ce discours sur les organisations internationales, voir par exemple United Nations Development Programme (UNDP), Governance for Peace: Securing the Social Contract.

2011 [en ligne], New York, UNDP, 2012

« http://www.undp.org/content/dam/undp/library/crisis\%20prevention/governance-for-peace_2011-1215_web.pdf.pdf.world development r2epo0rt 11» (consulté le 17 Janvier 2018).

17. On se réfère ici à la période qui va de la conclusion des accords de Linas-Marcoussis (2003) aux élections de 2010, caractérisée par des nombreuses négociations de paix et par une situation sécuritaire incertaine, où des moments de relative calme s'alternaient avec des explosions occasionnelles de violence. Pour une discussion plus approfondie, voir M. McGovern, Making War in Côte d'Ivoire, op. cit. 
(Onuci). La DAC était chargée de la mise en œuvre des Projets à Impact Rapide (Quick Impact Projects, QIP), qui visaient les besoins immédiats de la population, dans des secteurs tels que l'eau ou l'éducation, mais menait aussi des activités visant à « renforcer la cohésion sociale à la suite d'un dialogue intercommunautaire ${ }^{18}$ ». L'influence de l'approche "par le bas » était visible aussi dans d'autres programmes censés appuyer la mise en œuvre de l'Accord Politique de Ouagadougou, tels que le Projet d'Assistance Post Conflit (PAPC) cofinancé par la Banque Mondiale, qui adoptait explicitement l'approche de community driven development ${ }^{19}$.

Après 2011 le gouvernement ivoirien a commencé aussi à s’impliquer dans ce type d'activités. Le premier pas dans ce sens a été le lancement du Programme Présidentiel d'Urgence (PPU), conçu par le président et ses collaborateurs à partir de sa résidence temporaire au Golf Hôtel d'Abidjan ${ }^{20}$. Le programme suivait le modèle des QIP mis en œuvre auparavant par l'Onuci, avec des investissements dans le domaine de l'eau potable, de la santé, de l'éducation, de l'électricité, de la salubrité urbaine et de la réhabilitation des préfectures.

En 2012, le gouvernement ivoirien a fait un pas supplémentaire, décidant de créer un cadre commun pour gérer ses activités, si bien que celles des partenaires, dans le domaine de la réhabilitation et du dialogue communautaire, le PNCS (aujourd'hui rebaptisé Programme National de Réconciliation et de Cohésion Sociale (PNRCS)). Le mandat initial du PNCS, dont la gestion a été confiée à Mariatou Koné, professeure d'anthropologie à l'Université Félix Houphouët-Boigny, et ministre du gouvernement depuis 2017, allait de 2012 à 2015. Il a été par la suite prolongé jusqu'à 2020 et le $\operatorname{PNRCS}^{21}$ a reçu la mission additionnelle de se charger de l'indemnisation des victimes de guerre, en partenariat avec la nouvellement établie Commission Nationale pour la Réconciliation et l'Indemnisation des Victimes (Conariv).

Les vagues et ambitieux objectifs du PNCS/PNRCS sont de « promouvoir la cohésion sociale et la réconciliation nationale» et notamment "ressouder le tissu social, cultiver le vivre ensemble, créer les conditions d'une paix sociale durable et trouver des solutions durables aux grands problèmes qui mettent en péril la cohésion nationale ${ }^{22}$ ». Un volet du PNCS vise explicitement à financer des actions de promotion de la paix entre les communautés à la base. Selon les responsables du programme, ce sont les communautés, les leaders locaux et les citoyens ordinaires qui « doivent assurer, perpétuer les effets de la réalisation sur le terrain de façon permanente ${ }^{23}$ ». Une partie des activités du PNRCS est choisie en collaboration avec les partenaires internationaux, tels que l'ONUCI (en son temps) et le Programme des Nations Unies pour le Développement, ou des ONG Ivoiriennes, tels que West Africa Network for Peacebuilding-Côte d'Ivoire ${ }^{24}$. Selon les responsables, les autres activités sont à la fois choisies à partir des propositions provenant des acteurs locaux, ou initiées par le PNCS en réponse à des besoins urgents dans les zones les plus affectées par le conflit ${ }^{25}$.

18. Opération des Nations Unies en Côte d'Ivoire (ONUCI), « Affaires Civiles [en ligne] », Organisation des Nations Unies (ONU), <https://onuci.unmissions.org/affaires-civiles-0>, consulté le 25 Avril 2017.

19. Banque Mondial, Project Information Document (PID), Appraisal Stage: Emergency Post-Conflict

Assistance Project [en ligne], rapport n ${ }^{\circ}$ AB3187,

$<$ http://documents.banquemondiale.org/curated/fr/218321468770752305/Cote-dIvoire-Emergency-Post-

Conflict-Assistance-Project>, consulté le 25 Avril 2017.

20. République de Côte d’Ivoire, « Programme Présidentiel d’Urgence » [en ligne], 2012

<http://www.presidence.ci/mobile/presentation.php?i=85>, consulté le 11 Avril 2017.

21. Ministère de la Solidarité, de la Cohésion Sociale et de l'Indemnisation des Victimes, Programme National de Réconciliation et de Cohésion Sociale (PNRCS) 2016-2020, République de Côte d’Ivoire, Abidjan.

22. Ministère de la Solidarité, Programme National, op. cit.

23. Entretien avec responsables du PNCS, cit.

24. PNCS, Réalisations 2015 en Matière de Cohésion Sociale, de Réconciliation Nationale et d'Indemnisation des Victimes des Crises Survenues: Rapport General, Décembre 2015.

25. Entretien avec responsables du PNCS, cit. 
Depuis le lancement du PNRCS, la rhétorique de la cohésion sociale, définie comme « la clé et le moteur qui permettra de déclencher une nouvelle dynamique pour restaurer la confiance, la réconciliation et la paix ${ }^{26}{ }$, est devenue omniprésente, aussi bien parmi les décideurs internationaux que chez les Ivoiriens. Dans son premier discours de fin d'année après sa réélection en 2015, Ouattara mentionnait comme premier chantier pour la nouvelle année « la cohésion sociale et la réconciliation sans lesquelles tous nos efforts seront vains ${ }^{27}$ » tandis qu'en 2017 il affirmait, avec plus d'optimisme que la Côte d'Ivoire « a renoué avec la paix et la stabilité (...). Elle a retrouvé sa cohésion ${ }^{28}{ }^{2}$.

Ce consensus autour de la cohésion sociale masque cependant une série d'ambiguïtés. Notamment, dans le discours de la cohésion sociale, et dans les projets de réconciliation par le bas prôné par le PNRCS, le rôle de la politique nationale dans la production et la persistance de la fracture sociale est minimisé, comme je l'explique dans la section suivante, où j'analyse les caractères paradoxaux de la construction de la paix au niveau local en Côte d'Ivoire.

\section{Les ambiguïtés de la cohésion sociale en Côte d'Ivoire}

Les programmes de réhabilitation communautaire et de promotion de la cohésion sociale mises en place par le gouvernement ivoirien et par les partenaires au développement se basent sur une hypothèse implicite : il est possible de bâtir la cohésion sociale " par le bas » et de réconcilier des communautés de base dans un contexte dans lequel non pas un accord de paix, mais le déséquilibre de pouvoir à faveur d'un camp, est le fondement de la stabilité politique «par le haut ».

Il faut remarquer que l'insistance excessive sur les potentialités du niveau local est un aspect du discours international du local turn. Les partisans du local turn attribuent aux communautés une autonomie qu'elles ne possèdent pas nécessairement. Ils représentent de manière artificielle les niveaux national et local comme séparés, négligeant les importants liens entre paix locale et paix nationale ${ }^{29}$.

L'application à la Côte d'Ivoire du paradigme de la construction de la paix au niveau local est particulièrement problématique pour deux raisons. Premièrement, dans le contexte Ivoirien, les projets d'intervention au niveau local ne se sont pas développés comme un instrument pour donner un enracinement à l'échelle locale d'un processus de paix construit à l'échelle nationale, mais comme un effort de compensation du processus de paix non effectif au niveau national. Cette tendance, déjà visible dans la période 2007-2010, quand l'application des accords de paix trainait, est de plus en plus apparente dans le contexte post-victoire militaire de l’après 2011.

26. Le Coordonnateur humanitaire du Système des Nations Unies en Côte d’Ivoire, Ndolamb Ngokwey, cité par Xinhua, «La cohésion sociale est la 'clé' de la paix en Côte d’Ivoire (ONU)» [en ligne], Abidjan.net, 20 Aout 2013, <http://news.abidjan.net/h/469412.html>, consulté le 23 Décembre 2017.

27. Présidence de Côte d’Ivoire, Message à la Nation du Président de la République, S.E.M. Alassane Ouattara, du jeudi 31 Décembre 2015 [en ligne], 2 Janvier 2016 <http://www.presidence.ci/message-a-la-nation-dupresident-de-la-republique-s-e-m-alassane-ouattara-du-jeudi-31-decembre-2015/>, consulté le 23 Décembre 2017.

28. Alassane Ouattara, «Congrès du RDR: Les grandes lignes d'orientation d'Alassane Ouattara (Discours intégral)», Fraternité Matin [en ligne], <https:/www.fratmat.info/index.php/politique/3eme-congres-du-rdr-lesgrandes-lignes-d-orientation-d-alassane-ouattara-discours-integral>, consulté le 23 Décembre 2017.

29. Pour une critique du local turn, voir A. Ernstorfer, D. Chigas et H. Vaughan-Lee, « From Little to Large: When Does Peacebuilding Add Up? », Journal of Peacebuilding \& Development, vol. 10, n 1, 2015, p. 72-77; C. Simons, F. Zanker, A. Mehler et D.M. Tull, « Power-sharing in Africa’s War Zones: How Important is the Local Level? », The Journal of Modern African Studies, vol. 51, n 4, 2013, p. 681-706. 
Deuxièmement, les élites politiques ont joué historiquement un rôle décisif dans la polarisation de la société Ivoirienne ${ }^{30}$. La « fracture communautaire ${ }^{31}$ » ivoirienne découle en partie de la compétition entre différentes communautés autour de l'accès à la terre, dans un contexte de pression démographique et de crise économique, mais également de l'opposition des populations du Sud-Ouest à l'immigration historiquement encouragé par HouphouëtBoigny $^{32}$. Cependant, cela n'a pas atteint un niveau significatif de violence avant l'intensification de la compétition politique et l'introduction du multipartisme en $1990^{33}$. C'est l'exclusion de Alassane Ouattara du jeu politique, et les actes posés par les figures dominantes de la politique ivoirienne post-Houphouët-Boigny - Henri Konan Bédié, Laurent Gbagbo et Ouattara lui-même - qui ont fait monter la tension entre les communautés jusqu’à l'avènement de ce qu'un groupe de chercheurs ont pu qualifier d' " année terrible » $1999^{34}$ et de l'insurrection des Forces Nouvelles en 2002. En dépit des apparences, les élites politiques ont joué un rôle crucial dans l'émergence des formes de mobilisation potentiellement violente présentées comme des initiatives «spontanées" de la population à la base, tel que le mouvement des « Jeunes Patriotes », l'un des soutiens du régime Gbagbo ${ }^{35}$.

Si on regarde les documents de programmation du PNRCS, les aspects problématiques d'une paix acquise par la défaite du camp adverse sont complètement négligés. Par rapport à la situation politique de l'après 2011, l’on affirme simplement que «la légalité constitutionnelle a été restaurée en avril 2011», tandis que le programme se donne pour mission «la paix et de l'allègement des tensions dans les relations entre les différentes communautés» où bien «la paix et la concorde entre les populations». ${ }^{36}$ Il n’y a pas de stratégie de long terme, ni dans le cadre de ce programme ni dans l'action plus générale du gouvernement Ivoirien, à propos des grands questions qui paralysent la réconciliation au niveau nationale. La justice transitionnelle était approchée de façon apolitique et technocratique par le PNRCS et par son partenaire, la Conariv. Même si la Conariv avait une composition et structure très similaire à la CDVR, son mandat était beaucoup plus étroit et consistait à enregistrer les victimes et déterminer l'entité des réparations économiques. La nécessité de consolider la démocratie et restaurer la confiance de toutes les parties dans le jeu électoral reçoivent aussi peu d'attention dans le cadre du PNRCS et de la part du gouvernement Ivoirien plus en général. Par exemple, le PNRCS affirme que "les élections législatives de décembre 2011 et locales couplées (régionales et municipales) d’avril 2013, ainsi que l'élection présidentielle de décembre 2015 qui s’est déroulée dans le calme, ont parachevé le processus de remise en fonctionnement des institutions de la République ${ }^{37}$ » et il ne fait pas mention des problèmes de boycott de l'opposition et de manque de confiance dans les institutions électorale qui ont affecté ces élections.

30. Voir par example A. Langer, « Horizontal inequalities and violent conflict: the case of Côte d'Ivoire», Working Paper $\mathrm{n}^{\circ}$ 13, Centre for Research on Inequality, Human Security and Ethnicity (CRISE), University of Oxford, 2004; T. Coulibaly, «Démocratie et surenchères identitaires en Côte d’Ivoire», Politique Africaine, $\mathrm{n}^{\circ}$ 58, 1996, pp. 143-150; F. Akindès, «The roots of the military-political crises», op. cit.

31. O. Dembele, « Côte d'Ivoire: la fracture communautaire », Politique africaine, ${ }^{\circ}{ }^{89}$, 2003, pp. 34-48. 32. F. Akindès, «The roots of the military-political crises», op. cit.; R. Marshall Fratani, The War of «Who Is Who»: Autochthony, Nationalism, and Citizenship in the Ivorian Crisis, «African Studies Review», vol. 49, $\mathrm{n}^{\circ}$ 2, 2006, pp. 9-43.

33. O. Dembele, «Côte d'Ivoire: la fracture communautaire», art. cité

34. M. Le Pape et C. Vidal, Côte d'Ivoire: l'année terrible 1999-2000, Paris, Karthala, 2002.

35. G. Koné, Les «Jeunes Patriotes»: ou la revanche des «porteurs de chaises» en Côte d'Ivoire, Abidjan, Les Classiques Ivoiriens, 2014 ; G. Piccolino, « David against Goliath in Côte d’Ivoire? Laurent Gbagbo’s War against Global Governance», African Affairs, vol. 111, nº. 442, 2012, pp. 1-23.

${ }^{36}$ Ministère de la Solidarité, Programme National de Réconciliation et de Cohésion Sociale (PNRCS) 20162020, cit., Introduction, p.5. Italique par l’autrice de cet article.

${ }^{37}$ Ministère de la Solidarité, Programme National de Réconciliation et de Cohésion Sociale (PNRCS) 20162020, cit., pp.9-10. 
L'on relève de contradictions aussi dans le discours du gouvernement ivoirien sur la construction de la paix au niveau local. D’un côté, reprenant la rhétorique Houphouétiste, la Côte d'Ivoire est représentée comme un pays essentiellement harmonieux et pacifique, dans lequel « il n’y a pas de véritable animositée ${ }^{8}$ » entre les citoyens ordinaires. De l'autre côté, l'on insiste sur l'idée que ce sont ces citoyens ordinaires qui ont la responsabilité principale de se réconcilier entre eux : «Votre propre voisin du quartier, avec lequel vous êtes en palabre, ce n'est pas le député, ce n'est pas le maire, c'est le commerçant, c'est l'artisan, c'est le chauffeur, avec lequel vous vivez. Donc, c'est avec lui que vous devez vous réconcilier d'abord $^{39}$ ".

La position du gouvernement ivoirien devient plus compréhensible si l'on considère que, comme le reconnaissent aussi les partenaires internationaux, ces interventions à l'échelle locale visent aussi à « établir sa légitimité au milieu d'une population divisée ${ }^{40}$ ». Le PPU était clairement formulé pour marquer politiquement l'engagement personnel de Ouattara dans la reconstruction du pays ${ }^{41}$. De façon moins directe, le PNRCS vise le même objectif. Il apparait aussi comme un moyen pour le régime Ouattara d'incrémenter sa popularité ${ }^{2}$.et participe à la philosophie globale de construction de la paix du même régime. A l'apparence, le PNRCS contredise l'idée que le développement économique puisse automatiquement amener la paix, car il prévoit des actions spécifiques au-delà de la politique économique à mettre en œuvre pour aider la Cote d'Ivoire à sortir de la période de crise. En réalité il remplit aussi la fonction de déplacement de l'attention du niveau des grandes questions de politique nationale, au niveau des victimes et des communautés.

De cette façon, le glissement de rhétorique, de la « réconciliation nationale » à la « cohésion sociale ", marque une dépolitisation de la construction de la paix. Comme un responsable du PNRCS le souligne, la différence entre les deux est que le concept de cohésion sociale n'est pas forcement lié à un conflit passé : "Quand il y a réconciliation, c'est bien qu'il y a eu un conflit. Alors que la cohésion sociale, c'est le fait de vivre en harmonie et la cohésion sociale est une quête permanente ${ }^{43}$ ".

Dans la prochaine section, je montre d'une part que cette vision de la " cohésion sociale » ne répond pas aux attentes des Ivoiriens, surtout des anciens électeurs de Laurent Gbagbo qui se sentent marginalisés dans le contexte actuel ; d'autre part, que le manque de réconciliation et de compromis « par le haut » affecte la construction de la paix « par le bas ».

\section{Le point de vue des citoyens « du bas »}

Comment les Ivoiriens voient-il cette offre politique de construction de la paix ? Quels sont les sources de blocage qu'ils perçoivent dans le processus de construction de la paix?

38. Entretien avec responsables du PNCS, cit.

39. Entretien avec responsables du PNCS, cit.

40. Banque Mondiale, Restructuring Paper on a Proposed Project Restructuring of the Post Conflict Assistance Project, Grant July 2, 2007 to the Republic of Côte d'Ivoire » [en ligne], Sustainable Development Department, Country Department AFCF2, Africa Region, 6 Octobre 2011

<http://projects.worldbank.org/P082817/emergency-post-conflict-assistance-project?lang=en> , consulté le 1 Mai 2017, traduit de l’Anglais par l'autrice.

41. La Lettre du Continent, « Bonus pour le Programme présidentiel d'urgence », 22 Octobre 2014.

${ }^{42}$ Ce n’est pas un hasard que Mariatou Koné ait été nommée ministre en 2016, pour donner plus de visibilité au PNRCS et à son étroite relation avec les acteurs politiques

43. Entretien avec responsables du PNCS, cit. 
Le matériel présenté ici est issu d'une série de focus groups conduits avec des Ivoiriens appartenant à plusieurs couches sociales ${ }^{44}$. Ces personnes sont exposés à la rhétorique du gouvernement qui incite à bâtir la cohésion sociale. Certaines d'entre elles ont eu des expériences de participation à des programmes de dialogue communautaire ou sont affiliées à des associations locales qui, dans le contexte actuel, ont été la cible d'interventions de promotion de la cohésion sociale.

Pour renseigner ces problématiques, nous avons généralement commencé les entretiens en demandant aux personnes interrogées de donner leur définition de la paix et de discuter comment, à leur avis, on peut construire la paix en Côte d'Ivoire. Nous leur avons ensuite posé des questions sur la vie quotidienne dans leurs quartiers et villages et sur les défis principaux auxquels elles se trouvent confrontées après 2011. Dans certains cas, la conversation a spontanément viré sur la politique nationale et l'attitude du gouvernement ivoirien. Dans d'autres, nous avons demandé explicitement aux personnes interrogées ce qu'ils pensaient des initiatives du gouvernement visant à soutenir la réconciliation et la cohésion sociale, tels que la CDVR, le PNCS et le programme de Désarmement, Démobilisation et Réintégration (DDR).

Plusieurs observations se dégagent de ces entretiens. Premièrement, la paix au niveau local est une préoccupation effective pour un bon nombre d'Ivoiriens, qui sentent que, même si les relations entre communauté se sont améliorées depuis la fin de la crise, la méfiance et des antagonismes persistent. Les clivages ethniques continuent à structurer la société Ivoirienne, avec d’un côté les « Nordistes » (Malinké, Sénoufo, migrants Burkinabés, etc.) identifiés automatiquement comme pro-Ouattara et de l'autre, les "autochtones de l'Ouest» - les citoyens appartenant aux groupes linguistiques Krou (Bété, Guéré, etc.) et certains groupes Mandé du Sud (Gouro, etc.) perçus ou se définissant comme pro-Gbagbo. Dans ce sens, on peut dire que les efforts de construction de la paix au niveau local répondent effectivement à une demande des populations. Cependant, pour beaucoup de personnes interrogées, la racine des problèmes se trouve dans la situation politique au niveau national.

Deux mécanismes reliant la situation locale et celle nationale peuvent être identifiées. D’un côté, de façon active, les cadres politiques exercent une influence souvent négative sur les communautés, particulièrement en période électorale. De l'autre côté, de manière plus générale, le fait que la paix au niveau national n'est pas basée sur un compromis entre les camps Ouattara et Gbagbo, mais sur la victoire d'un camp sur l'autre, et les injustices avérées

\footnotetext{
${ }^{44}$ Nos collaborateurs et nous avons conduit vingt focus groups entre Mai et Juillet 2015, dont huit à Abidjan (dans les quartiers de Abobo, Yopougon, Adjamé et Cocody) et douze dans le Sud-Ouest (quatre à Daloa, commune et sous-préfecture, quatre à Duékoué et quatre à Gagnoa). Les répondants sont des Ivoiriens appartenant à plusieurs couches sociales - femmes de marché, leaders d'associations communautaires, transporteurs, étudiants, enseignants d'école - ainsi que certaines catégories de personnes particulièrement impliquées dans la crise - anciens combattants et anciens déplacés. Au total, huit entretiens ont été conduits avec des groupes de personnes issues des quartiers ou villages avec une population majoritairement favorables à l'ancien président Gbagbo, sept avec des personnes issues de communautés proches du RHDP et cinq avec des groupes mixtes.

Les personnes interrogées ont été recrutées à partir de contacts différents. Dans certains cas, nous sommes passés par le biais des organisations de quartier ou d'associations de femmes, dont certaines avaient travaillé avec des partenaires internationaux, notamment Search for Common Ground (SFCG). L'implication de SFCG a été un passage obligé dans les terrains plus sensibles, tels que les entretiens avec les anciens combattants ou dans les quartiers d'Abidjan théâtres de violence, où le fait que les interlocuteurs avaient déjà établi une relation de confiance avec l'ONG a aidé à surmonter leurs méfiances initiales. Dans d'autres cas, notamment à Daloa et Gagnoa, nous nous sommes adressés à la commune et à la sous-préfecture, qui nous a mis en contact avec des coopératives et associations locales et avec des chefs de village. Dans d'autres cas encore, des contacts personnels dans le milieu scolaire et universitaire et des organisations professionnelles (i.e. syndicats de transporteurs ou d'enseignants) nous ont fourni un point d'accès. De façon générale, nous avons essayé de ne pas utiliser le même mécanisme pour recruter les différents groupes, afin de minimiser le risque de biais lié aux modalités du recrutement.
} 
ou présumées qui en découlent, sont une source de mécontentement parmi les anciens électeurs de Gbagbo. Il est important de faire remarquer que la provision de biens publics par le gouvernement Ouattara dans cette partie du pays semble avoir un impact très limité sur l'attitude de ces populations vis-à-vis à la paix et à leur perception des actions du gouvernement.

\section{La paix locale et les problèmes actuels}

L’importance donnée par le gouvernement ivoirien et par les bailleurs de fond à la cohésion sociale n'est pas en soi même inappropriée. Nos entretiens confirment d'un côté l'importance que les Ivoiriens accordent à la dimension quotidienne de la paix (« la paix c'est le vivre ensemble heureux, dans l'harmonie et s'entendre ${ }^{45} » \ldots$ « « c'est l'entente entre les différentes populations, la tranquillité et puis, être en union parfaite $\left.{ }^{46} »\right)$ et le fait qu'aux yeux de la plupart des Ivoiriens, cette paix n'est cependant que partiellement acquise, malgré la cessation des violences. Dans les quartiers auparavant affectés par la violence « les gens font semblant. Ils font comme si ça va... le conflit est au stade latent ${ }^{47}$ ». Le mot le plus utilisé par les personnes interrogées pour décrire la situation actuelle est « méfiance ${ }^{48}$. Une forme dans laquelle les tensions se manifestent est celle qu'une de nos interlocuteurs qualifie de "violence verbale ${ }^{49}$ ». Il s'agit de remarques de nature ethnique ou politique, parfois ouvertement hostiles, parfois déguisés en blague : "Souvent même, ces injures ne sont pas des injures directes, c'est 'espèce de Dioula là!'... souvent ils disent, 'partez avec votre président là 50 ». Seulement dans les villes du « Far Ouest » Ivoirien près de la frontière avec le Liberia (Duékoué, Guiglo) les interlocuteurs mentionnent des incidents violents. Cependant la méfiance et les antagonismes ethno-communautaires voilés sont répandus dans toutes les régions de la Côte d’Ivoire visitées.

\section{Le rôle de la politique nationale}

Les citoyens expriment très souvent l'opinion que « la politique » est la cause centrale des divisions entre les citoyens. Selon un habitant d'un quartier d'Abidjan considéré pro-Ouattara « la politique, c'est celle-là qui nous détruit, qui nous divise. Parfois les gens en arrivent aux mains ${ }^{51}$ ». Selon une femme qui travaille au marché d'Abobo «beaucoup de personnes ont peur de s'exprimer à cause de la politique qui fait du mal à l'Afrique ${ }^{52}$ ". Les élections sont une cause de peur et anxiété ${ }^{53}$. Les politiciens sont accusés d'un côté d'instrumentaliser les sentiments ethniques. Le politicien est vu comme «celui qui vient dire toi tu es Dioula, le Bété dit que tu es mauvais, le Bété dit que tu n’es pas d'ici ${ }^{54} »$. De l'autre côté, ils sont aussi

45. Focus group, femmes de marché dans le quartier de Abobo, Association des Femmes la solidarité, Abobo, Abidjan, 20 Mai 2015.

46. Focus group, jeunes du quartier de Yao Sehi, Comité de Coordination de la Société Civile de Youpougon, Yopougon, Abidjan, 21 Mai 2015.

47. Focus group, Yao Sehi, cit.

48. Focus group, représentants de syndicats estudiantins, Université Félix Houphouët-Boigny, campus de Cocody, Abidjan, 27 Mai 2015. Focus group, représentantes d'associations de femmes autochtones, Bureau de la Sous-préfecture, Gagnoa, 13 Juillet 2015.

49. Focus group, Fédération des Associations des Femmes de la région du Bas Sassandra, Mairie de Daloa, Daloa, 17 Juin 2015.

50. Focus group, Fédération des Associations des Femmes de la région du Bas Sassandra, cit.

51. Focus group, jeunes du quartier de Doucouré, Comité de Coordination de la Societé Civile de Youpougon, Youpougon, Abidjan, 21 Mai 2015.

52. Focus group, femmes de marché, cit.

53. Focus group, femmes de marché, cit.

54. Focus group, Doucouré, cit. 
accusés de profiter de la pauvreté et du manque d'emploi pour recruter les jeunes dans une militance potentiellement violente ${ }^{55}$.

Dans les entretiens conduits à Abidjan, les interlocuteurs apparaissent souvent conscients et critiques par rapport à la manipulation des politiciens. Ils font aussi plus souvent référence aux besoins économiques comme facteur qui pousse les gens ordinaires à suivre les politiciens. Par exemple, pour une femme animatrice d'une association de quartier, la sécurité économique « est très importante pour la paix parce que, si tu es bien logé, tu n'as pas besoin d'aller rester dans salon de quelqu'un. Si tu es dans salon de quelqu'un, tu dors là-bas, tu es obligé de faire ce que lui, il veut ${ }^{56}$ ». Pour une autre femme, commerçante, "si les gens mangent normalement, il n’y aura pas palabres... C’est la pauvreté. Donc la partie qui vient te donner vingt-mille francs, tu vas la suivre. C'est ça qui fait que la guerre là est là ${ }^{57}$ ». Dans les entretiens conduits à l'Ouest, cette conscience politique apparait moins explicite. Dans ces zones, les politiciens semblent exercer une plus forte emprise sur la population: pendant notre recherche, nous avons été impressionnés par le fait que des planteurs et villageois reproduisent souvent les mêmes discours et utilisent les mêmes mots que des politiciens proGbagbo. Les politiciens sont consultés par les populations et utilisent d'ailleurs souvent leur influence pour entraver les projets de promotion de la "cohésion sociale». Selon le responsable d'une ONG, " vous partez dans une localité faire la sensibilisation, dès que vous tournez le dos, on prend le téléphone pour appeler le cadre du village ${ }^{58} »$.

Contredisant le discours du PNRCS, certains Ivoiriens insistent sur le fait que les leaders politiques ont le devoir d'initier l'effort pour la paix. Selon un jeune d'un quartier Abidjanais considéré pro-Gbagbo « c'est eux qui sont à la tête, s'ils ne s'entendent pas il ne peut pas avoir la paix. C'est par eux d'abord que tout commence ${ }^{59}$ ». Un villageois de la région de Gagnoa énonce une opinion similaire : " il y a deux personnes qui sont en conflit [Ouattara et Gbagbo, n.d.a.], dès que les deux se réconcilient là, tous ceux qui sont derrière eux, c'est fini, c'est à dire on n'en parlera même pas ${ }^{60}$ ». Certains interlocuteurs, cependant, même en étant critiques de l'attitude des politiciens, soulignent que la population doit aussi prendre ses responsabilités : «La paix c'est entre nous d'abord, nous les populations. Les hommes politiques, ils viennent nous inciter à la chose [la violence, n.d.a], mais il suffit de la bonne compréhension, on va vivre en parfaite harmonie ${ }^{61}$ ». Même si ce genre de discours rappelle la position du PNCS, il faut observer que l'accent n'est pas mis sur la réconciliation entre communautés en tant que telles, mais sur la capacité des citoyens " d'en bas » à s'organiser pour résister aux gens « d'en haut ». On veut « une cohésion qui nous rendra forts face au politiciens $^{62} »$.

\section{Les revendications des communautés du Sud-Ouest}

Le « camp Gbagbo » a des doléances spécifiques, qui ont leurs origines dans la situation de déséquilibre de pouvoir au niveau national, mais ont aussi des répercussions concrètes au niveau local. Il y a parmi les «autochtones" de l'Ouest un sentiment général que les communautés associées aux RHDP, surtout les communautés du Nord, sont favorisées dans

55. Focus group, femmes de marché, cit.

56. Focus group, représentantes d'associations de femmes, Centre Sociale de Abobo, Abobo, Abidjan, 19 Mai 2015.

57. Focus group, femmes de marché, cit.

58. Focus group, ancien déplacés internes, Siège de l’ONG Bonne Action, Duékoué, 30 Juin 2015

59. Focus group, Yao Sehi, cit.

60. Focus group, planteurs and villageois, place publique, Village de Seria, sous-préfecture de Daloa, 27 Juin 2015.

61. Focus group, Yao Sehi, cit.

62. Focus group, Yao Sehi, cit. 
l'accès à l'emploi public et aux ressources ${ }^{63}$. Cependant, le sens de mécontentement des anciens électeurs de Gbagbo semble aussi découler de questions qui n'affectent pas de façon directe les personnes interrogées. Surtout dans les régions de l'Ouest, la question de la libération de Laurent Gbagbo et des autres personnalités liées à l'ancien régime qui sont en prison (qualifiés, à tort ou raison, des prisonniers politiques) est évoquée comme une des principales doléances : «Ceux qui sont en exil, ceux qui sont emprisonnés, tous ceux-là si on les libère, pour venir juger, pour dialoguer, pour se parler, et on voit ce qu'y a eu... et puis on laisse passer tout ça là, la paix revient, y’a pas de problème... si ceux-là sont libérés, c'est fini $^{64}$ ». Plusieurs interlocuteurs soulignent le lien entre la question des prisonniers et les tensions entre communautés : « [Il y a] La violence verbale parce que, étant sur le terrain, la population surtout, demande la libération des prisonniers politiques. C'est ça même le gros problème $^{65} » \ldots$ « je ne peux pas aller me battre avec ceux qui sont à Abidjan. C'est mon frère RHDP qui est en face, dont les responsables ont mis mon frère en prison, c'est lui qui devient automatiquement mon ennemi ${ }^{66}$ ». De toute évidence, les discours sur la question des « frères en prison » au niveau communautaire reproduisent de façon presque identique le discours que le « FPI Sangaré » relate au niveau national.

\section{Paix par le développement?}

En prônant la "paix par le développement », le gouvernement ivoirien espère calmer ces sentiments à travers ses actions de développement local. La réhabilitation des infrastructures locales a été aussi une marque des programmes « construction de la paix » de l'Onuci et de la Banque Mondiale. Cependant, la teneur des entretiens ont permis de constater combien l'impact de ces actions sur la construction de la paix est limité, surtout dans les zones rurales. Un cas emblématique est celui de Gagnoa, ville considérée comme étant le fief de Laurent Gbagbo, où le PPU a réhabilité plusieurs bâtiments publics et terminé, en partenariat avec la coopération chinoise, la construction d'un nouvel hôpital. La perception de cet effort d'investissement public et de ces acquis est variable selon l'appartenance ethnique et politique des participants. Interrogées les indicateurs du développement de la ville, un groupe de femmes de communautés originaires du Nord liste une longue série de travaux de réhabilitation (plusieurs routes, la préfecture, une banque, les bureaux du trésor, des impôts, et la Compagnie Ivoirienne d'Electricité) ${ }^{67}$. Par contre, la même question posée à un groupe de femmes d'ethnie bété ne donne pas le même résultat. Les femmes bété minimisent plutôt la portée de l'engagement du PPU, soulignent par exemple que la construction de l'hôpital avait été entamée par le gouvernement précédent, et ont tendance à mettre plutôt l'accent sur ce qui n’a pas été fait (« chez nous [dans les zones rurales] s’il pleut, y’a pas route ».... « les écoles d'ici sont décoiffées »). Et, après avoir admis à peine que la préfecture a été réhabilitée, terminent en disant : « sinon rien n’est fait à Gagnoa ${ }^{68}$ ».

Il est difficile de cerner la raison exacte de ce manque d'intérêt pour les projets développementalistes du régime Ouattara. La perception selon laquelle le support gouvernemental est disproportionnellement alloué aux « Nordistes » peut avoir joué un rôle.

63. Focus group, Yao Sehi, cit.

64. Focus group, représentantes d'associations de femmes autochtones, cit.

65. Focus group, Fédération des Associations des Femmes de la région du Bas Sassandra, cit.

66. Focus group, représentants d'organisations des populations autochtones, Restaurant « Le Talier du chef », Gagnoa, 11 Juillet 2015.

67. Focus group, représentants d'associations de femmes allogènes, Bureau de la Sous-préfecture, Gagnoa,13 Juillet 2015.

68. Focus group, représentantes d’associations de femmes autochtones, Bureau de la Sous-préfecture, Gagnoa,13 Juillet 2015. 
L’idée que l'économie puisse "guérir » les Ivoiriens semble donc présenter des limites intrinsèques. Car, certaines des revendications exprimées par l'élite politique pro-Gbagbo, et appropriées par les communautés, telles que la question des prisonniers politiques, ne sont pas de nature socio-économique mais plutôt identitaire et symbolique. Par exemple, pour une veuve d'ethnie guéré, ancienne déplacée, le soutien qu'elle a obtenu par le PAPC, programme géré par la primature Ivoirienne, apparait secondaire par rapport à la question des détenus politiques : c'est cela qui est « notre problème à nous tous ${ }^{69}$ ".

Il ressort clairement de ces entretiens avec les communautés « autochtones » de l'Ouest que ces dernières considèrent le régime Ouattara régime " Nordiste » et non légitime qui tient leurs « frères » ethniques en prison. La provision de biens publics n’a pas permis de changer une telle perception dominante.

\section{Conclusion: les raisons de la stabilité et la promotion de la paix au niveau locale}

Cette contribution se veut une analyse de certains aspects du modèle de reconstruction postconflit prôné par le gouvernement Ivoirien, et, au même temps, une critique du local turn qui, aux yeux de certains chercheurs et praticiens, est devenu le nouveau «bâton magique » des programmes de construction de la paix. L’idée que la voie pour bâtir la paix dans des sociétés divisées soit celle de la prise en compte du niveau local et communautaire semble attractive. Cependant, dans un contexte comme celui de la Côte d'Ivoire de l'après conflit, les divisions parmi la population sont intrinsèquement liées à la situation de " paix des vainqueurs » dans laquelle le pays se trouve. Les instruments que le gouvernement Ivoirien et la communauté internationale entendent utiliser pour bâtir la « cohésion sociale » semblent ne pas changer la donne de façon significative.

Sous certains aspects, la situation actuelle rappelle l'inversion de celle de 2002 mais avec quelques différences. Au sein des communautés ethniques qui ont auparavant soutenu Gbagbo, l'élite politique de la «branche dissidente » du FPI encourage des sentiments - en partie justifiés - de marginalisation et discrimination. Mais l'élite pro-Gbagbo est affaiblie par la répression étatique et par ses divisions internes et ne dispose ni ressource politique, ni ressources militaires et économiques pour initier un nouveau conflit armé. Mais en même temps, l'on note d'importants clivages entre les différentes composantes de l'élite au pouvoir. Le RHDP doit régler la question de la succession de Ouattara: la compétition est en train de s'intensifier au sein des clans politiques composant cette coalition. Guillaume Soro et les militaires issus des anciennes Forces Nouvelles ont peur de perdre leurs actuelles positions de privilège. Ces derniers acteurs ont accès à des ressources militaires et économiques importantes, ce qui le rend potentiellement capables d'actions violentes. Mais ces hommes politiques n'ont pas la possibilité de mobiliser facilement les communautés autochtones de l'Ouest, hostiles à la coalition gouvernementale. Cette configuration de divisions sociopolitiques et de relations de pouvoir produit une situation de relative stabilité, dans laquelle le mécontentement de certaines couches de la population ne présente pas de risque de violence sur le court terme.

Cette contribution ne vise pas à nier l'intrinsèque valeur de certaines actions visant à construire la paix entre communautés. Mais il veut exprimer à la fois un certain scepticisme à propos de l'idée de « réconciliation par des matchs de football» et une certaine méfiance à propos du discours du gouvernement Ivoirien sur la promotion de la " cohésion sociale » entre communautés de base.

69. Focus group, ancien déplacés internes, Siège de l’ONG Bonne Action, Duékoué, 30 Juin 2015. 